\title{
TRAINING DESIGN IN SINGLE RELAY AF COOPERATIVE SYSTEMS WITH CORRELATED CHANNELS
}

\author{
Christos Mavrokefalidis ${ }^{\dagger}$, Athanasios A. Rontogiannis*, Kostas Berberidis ${ }^{\dagger}$ \\ ${ }^{\dagger}$ Dept. of Computer engineering and Informatics, University of Patras, Greece \\ Emails: \{maurokef, berberid\} @ ceid.upatras.gr \\ *Institute for Space Applications and Remote Sensing, National Observatory of Athens, Greece \\ Email: tronto@space.noa.gr
}

\begin{abstract}
In this paper, training design is studied for a single relay amplify-and-forward cooperative network. The taps of the frequency selective channels are assumed to be correlated and OFDM modulation is used for transmission. Based on the least squares (LS) criterion, conditions for pilot tone positioning and a number of power allocation schemes are described. Analytical closed-form power allocation expressions are provided for both the source and the relay. The theoretical results are fully corroborated by simulations.
\end{abstract}

Index Terms - Training design, LS criterion, correlated channels, cooperative communications

\section{INTRODUCTION}

Cooperative communication systems have attracted a lot of attention recently [1], due to their ability to exploit spatial diversity by utilizing relays to assist transmission between a source and a destination. One of the most popular transmission protocols in cooperative systems is the so-called amplifyand-forward (AF) scheme, in which the relay amplifies and forwards the received signal to the destination. In this paper, we are dealing with the problem of training design for channel estimation in a single relay AF system. All channels in the network are assumed frequency selective, while orthogonal frequency division multiplexing (OFDM) modulation is employed for transmission.

The design of the optimal training in relay based systems has gained increasing interest lately. More specifically, in $[2,3,4]$, optimal training design conditions were derived, while a single fixed gain amplification factor per relay was applied independently of the optimization procedure. In [5], different amplification factors per pilot tone (of the OFDM symbol) were incorporated in the design problem. However, in the suggested solutions, all these factors are proved to be

This work was supported by the 03ED838 research project, implemented within the framework of the "Reinforcement Programme of Human Research Manpower" (PENED) and co-financed by National and EC Funds.

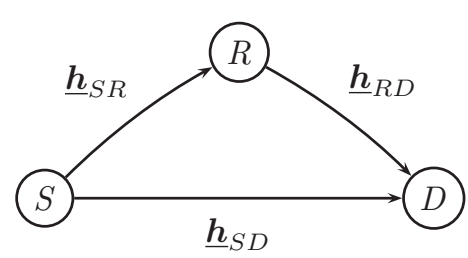

Fig. 1. The cooperative system

equal. All the previous works assume that the channels to be estimated, are uncorrelated. Space channel correlation was assumed in [6], where by using an LMMSE estimator, the optimal training design along with the amplification factors per pilot tone were derived through a convex optimization problem. However, since no closed-form expressions could be extracted, the correlation assumption was relaxed and a suboptimal solution was described. By experiments, it was shown that performance degradation could be considered negligible.

In this paper, the least-squares (LS) criterion is used and the minimum required number of pilot tones are employed. Under a general tap correlation model, conditions are derived for power allocation for the training symbols and their positioning at the source side, as well as power allocation for forwarding the pilot tones at the relay side. It is shown that the well-known conditions for pilot positioning (e.g. [7]) are independent of channel correlation. Thus, the general design problem is cast to a power allocation one, in which allocation of equal power to all pilots is not optimal. Although, the optimal solution to this problem can not be obtained in closedform, some interesting closed-form suboptimal power allocation solutions are provided, along with their corresponding mean squared error (MSE) performance, which are fully supported by experiments. From these closed-form expressions, the case of uncorrelated channels comes out naturally as a special case.

The outline of the paper is as follows. In Section 2, the system model is presented. Different training design schemes are presented and evaluated in Section 3. In Section 4, a discussion on the theoretical results along with simulations are presented to conclude the paper.

In the following, bold underlined small and capital letters 
denote vectors at the time and frequency domain, respectively. Also, bold capital letters are used for matrices. $\boldsymbol{F}$ denotes the $N \times N$ Fourier matrix whose $(p, q)$-th element is given by $[\boldsymbol{F}]_{p, q}=\frac{1}{\sqrt{N}} e^{-j 2 \pi p q / N} \cdot \boldsymbol{A}^{T}$ and $\boldsymbol{A}^{H}$ denote transposition and conjugate transposition of $\boldsymbol{A}$. Also, $\operatorname{diag}\{\underline{\boldsymbol{a}}\}$ produces a diagonal matrix with $\underline{\boldsymbol{a}}$ on its main diagonal and vect $\{\boldsymbol{A}\}$ produces a vector whose elements are the diagonal elements of $\boldsymbol{A} . \quad \boldsymbol{I}_{N}$ is the identity matrix of size $N, \operatorname{Tr}\{\boldsymbol{A}\}$ is the trace of $\boldsymbol{A}$ and $\mathcal{E}\{$.$\} denotes statistical expectation. Finally,$ $\underline{\boldsymbol{x}} \sim C N(\boldsymbol{\mu}, \boldsymbol{\Sigma})$ denotes a complex Gaussian random vector with mean $\bar{\mu}$ and covariance matrix $\boldsymbol{\Sigma}$.

\section{SYSTEM MODEL}

In Fig. 1, the frequency selective channels $\underline{\boldsymbol{h}}_{S D}, \underline{\boldsymbol{h}}_{S R}$ and $\underline{\boldsymbol{h}}_{R D}$ are modeled as vectors of lengths $L_{S D}, L_{S R}$ and $L_{R D}$, respectively. Also, it is assumed that $\underline{\boldsymbol{h}}_{i} \sim C N\left\{\underline{\mathbf{0}}, \boldsymbol{C}_{i}\right\}$ and $C_{i}$ is the correlation matrix of channel $i$, where $i \in$ $\{S D, S R, R D\}$. Transmission in the network is performed in blocks of $N$ symbols by utilizing OFDM and " $S$ ", " $R$ " are assumed to be synchronized. To avoid interblock interference, a cyclic prefix (CP) of appropriate length is appended to the transmitted signals ([8]), however, this issue will not be elaborated any further.

A two-phase non-orthogonal transmission protocol [9] is used by " $S$ " in order to transmit information to " $D$ ". At the first phase, " $S$ " sends the OFDM symbol $\underline{\boldsymbol{x}}_{1}=\boldsymbol{F}^{H} \underline{\boldsymbol{X}}_{1}$ at both " $R$ " and " $D$ " in which the received signals are

$$
\begin{gathered}
\underline{\boldsymbol{y}}_{R}=\boldsymbol{H}_{S R} \underline{\boldsymbol{x}}_{1}+\underline{\boldsymbol{w}}_{R}, \\
\underline{\boldsymbol{y}}_{1}=\boldsymbol{H}_{S D} \underline{\boldsymbol{x}}_{1}+\underline{\boldsymbol{w}}_{1},
\end{gathered}
$$

respectively. At the second phase, " $S$ " transmits a new symbol $\underline{\boldsymbol{x}}_{2}=\boldsymbol{F}^{H} \underline{\boldsymbol{X}}_{2}$ and, concurrently, " $R$ " amplifies and forwards to " $D$ " the symbol received at the previous phase. The received signal in this case is given by

$$
\underline{\boldsymbol{y}}_{2}=\boldsymbol{H}_{S D} \underline{\boldsymbol{x}}_{2}+\boldsymbol{H}_{R D} \boldsymbol{K} \underline{\boldsymbol{y}}_{R}+\underline{\boldsymbol{w}}_{2} \text {. }
$$

In (1)-(3), $\underline{\boldsymbol{w}}_{R} \sim C N\left(\mathbf{0}, \sigma_{R}^{2} \boldsymbol{I}_{N}\right)$ and $\underline{\boldsymbol{w}}_{1}, \underline{\boldsymbol{w}}_{2} \sim C N\left(\mathbf{0}, \sigma_{D}^{2} \boldsymbol{I}_{N}\right)$ are the involved noise vectors. The $N \times N$ matrices $\boldsymbol{H}_{i}$ are circulant, having as first columns the vectors $\left[\underline{\boldsymbol{h}}_{i}^{T} 0 \ldots 0\right]^{T}$ and can be expressed as $\boldsymbol{H}_{i}=\boldsymbol{F}^{H} \boldsymbol{\Lambda}_{i} \boldsymbol{F}$, where the diagonal matrix $\Lambda_{i}$ contains the frequency response (i.e. DFT) of the channel $\underline{\boldsymbol{h}}_{i}$. We assume that the relay amplifies each OFDM tone with a different amplification factor by employing a diagonal matrix $\boldsymbol{A}$, to be defined shortly. This operation corresponds to pre-multiplication of $\underline{\boldsymbol{y}}_{R}$ with a circulant matrix $\boldsymbol{K}=\boldsymbol{F}^{H} \boldsymbol{A} \boldsymbol{F}$ in the time domain, as in (3).

In the following, we assume, without loss of generality, that $L_{S D}=L_{S R}+L_{R D}-1=L$ and a minimum number of $L$ tones in each $\underline{\boldsymbol{X}}_{i}$ are devoted to training. Also, let $\underline{\boldsymbol{h}}=$ $\left[\underline{\boldsymbol{h}}_{S D}^{T} \underline{\boldsymbol{h}}_{R}^{T}\right]^{T}$ be the vector of channels to be estimated by "D", where $\underline{\boldsymbol{h}}_{R}=\underline{\boldsymbol{h}}_{S R} * \underline{\boldsymbol{h}}_{R D}$ and $*$ denotes convolution.
After selecting $L$ out of $N$ pilot rows, from (2), (3), indexed by the set $\left\{i_{k}\right\}$, where $k=1,2 \ldots L$, the frequency domain vectors $\underline{\boldsymbol{Y}}_{i}=\boldsymbol{F} \underline{\boldsymbol{y}}_{i}, i=1,2$, can be expressed as [4]

$$
\underline{Y}=B \underline{h}+\underline{W}
$$

where $\underline{\boldsymbol{Y}}=\left[\underline{\boldsymbol{Y}}_{1, L}^{T} \underline{\boldsymbol{Y}}_{2, L}^{T}\right]^{T}, \underline{\boldsymbol{W}}=\left[\underline{\boldsymbol{W}}_{1, L}^{T} \underline{\boldsymbol{\boldsymbol { W }}}_{2, L}^{T}\right]^{T}$ and

$\boldsymbol{B}=\sqrt{N}\left[\begin{array}{lc}\boldsymbol{X}_{1, L} & \mathbf{0} \\ \boldsymbol{X}_{2, L} & \boldsymbol{A}_{L} \boldsymbol{X}_{1, L}\end{array}\right]\left[\begin{array}{cc}\boldsymbol{F}_{L} & \mathbf{0} \\ \mathbf{0} & \boldsymbol{F}_{L}\end{array}\right]=\sqrt{N} \boldsymbol{X}_{d}$

The meaning of subscript $L$ in the above expressions is that $L$ elements or rows $\left(\left\{i_{k}\right\}\right)$ have been retained from the respective vectors and matrices. It can be verified that $\underline{\boldsymbol{W}}_{1, L} \sim C N\left\{\mathbf{0}, \sigma_{D}^{2} \boldsymbol{I}_{L}\right\}$ and $\underline{\boldsymbol{W}}_{2, L}$ is zero mean with covariance matrix $\boldsymbol{C}_{L}=\sigma_{R}^{2} \boldsymbol{A}_{L} \boldsymbol{A}_{L}^{H} \boldsymbol{\Theta}_{R D, L}+\sigma_{D}^{2} \boldsymbol{I}_{L}$ and $\Theta_{R D, L}=\mathcal{E}\left\{\Lambda_{R D, L} \Lambda_{R D, L}^{H}\right\}$ is a diagonal matrix where the $k$-th diagonal entry is denoted by $\theta_{k, R D}^{2}$. It is noted that $\Theta_{R D, L}$ is not the correlation matrix of the OFDM frequency tones and that, in general, has different diagonal elements, i.e. $\theta_{k, R D}^{2}=\theta_{j, R D}^{2}, k \neq j$ ([10]). Also, $\boldsymbol{X}_{i, L}=\operatorname{diag}\left\{\underline{\boldsymbol{X}}_{i, L}\right\}$ $(i=1,2)$ and the $L \times L$ matrix $\boldsymbol{F}_{L}$ is produced by the corresponding $L$ rows of $\boldsymbol{F}$ and its first $L$ columns. In the above derivation, the relation $\underline{\boldsymbol{\Lambda}}_{i, L}=\sqrt{N} \boldsymbol{F}_{L} \underline{\boldsymbol{h}}_{i}, i \in\{S D, R\}$, was used ([7]), where $\underline{\boldsymbol{\Lambda}}_{i, L}=\operatorname{vect}\left\{\boldsymbol{\Lambda}_{i, L}\right\}$. Finally, the diagonal matrix $\boldsymbol{A}_{L}$ contains the $L$ amplification factors corresponding only to the pilot tones. Its $k$-th element is expressed as

$$
\alpha_{k}=\sqrt{\frac{e_{k}}{\theta_{k, S R}^{2} p_{k}+\sigma_{R}^{2}}},
$$

where $\theta_{k, S R}^{2}$ is the $k$-th diagonal element of $\Theta_{S R, L}$, which is defined similarly to $\boldsymbol{\Theta}_{R D, L} \cdot e_{k}$ is the mean energy assigned by the relay to the $k$-th pilot tone and $p_{k}$ is the energy assigned by " $S$ " to the $k$-th pilot symbol residing in position $i_{k}$ of $\underline{\boldsymbol{X}}_{1}$. For future use, we also define with $q_{k}$ the corresponding energy for the $k$-th pilot symbol in $\underline{\boldsymbol{X}}_{2}$.

It is assumed that the mean total energy available at the relay for forwarding pilot tones is $E_{R}$. It can be shown that this is achieved if $\sum_{k=1}^{L} e_{k}=E_{R}$. Also, " $S$ " assigns energies $\sum_{k=1}^{L} p_{k}=E_{1}$ and $\sum_{k=1}^{L} q_{k}=E_{2}$ to the first and second phase, respectively, and $E_{1}+E_{2}=E_{S}$ in total.

\section{TRAINING DESIGN FOR LS CHANNEL ESTIMATION}

The LS estimator of $\underline{\boldsymbol{h}}$ in (4) and the error covariance matrix $\boldsymbol{C}_{e}=\mathcal{E}\left\{(\underline{\hat{\boldsymbol{h}}}-\underline{\boldsymbol{h}})(\underline{\hat{\boldsymbol{h}}}-\underline{\boldsymbol{h}})^{H}\right\}$ that describes its performance, are given by [11]

$$
\underline{\hat{\boldsymbol{h}}}=\boldsymbol{B}^{-1} \underline{\boldsymbol{Y}}, \quad \boldsymbol{C}_{e}=\boldsymbol{B}^{-1} \boldsymbol{C}_{W} \boldsymbol{B}^{-H},
$$

where

$$
\boldsymbol{C}_{W}=\left[\begin{array}{cc}
\sigma_{D}^{2} \boldsymbol{I}_{L} & \mathbf{0} \\
\mathbf{0} & \boldsymbol{C}_{L}
\end{array}\right]
$$


In this section, the conditions for pilot tone positioning and power allocation will be determined. As it will be shown, the equispaced positioning and the zero power allocation in the second phase (i.e. $E_{2}=0$ ) are similar to results reported for the uncorrelated case, as in [4]. The design problem, then, will be formulated as a power allocation scheme with respect to $p_{k}$ 's and $e_{k}$ 's only.

The optimal training design can be determined by solving the following minimization problem

$$
\begin{aligned}
\min _{\underline{\boldsymbol{p}}, \underline{\boldsymbol{q}}, \underline{\boldsymbol{e}}, E_{1}, E_{2},\left\{i_{k}\right\}} \frac{1}{2 L} \operatorname{Tr}\left\{\boldsymbol{C}_{e}\right\} \\
\text { s.t. } \quad \sum_{k} e_{k}=E_{R} \\
\sum_{k} p_{k}=E_{1} \\
\sum_{k} q_{k}=E_{2} \\
E_{1}+E_{2}=E_{S},
\end{aligned}
$$

where the $L \times 1$ vectors $\boldsymbol{p}, \boldsymbol{q}$ and $\underline{\boldsymbol{e}}$ contain the parameters $p_{k}$, $q_{k}$ and $e_{k}$, respectively.

The minimization of (9) can be conducted based on the following lower bound expression.

$$
\begin{aligned}
M S E & = & \frac{1}{2 L} \operatorname{Tr}\left\{\boldsymbol{B}^{-1} \boldsymbol{C}_{W} \boldsymbol{B}^{-H}\right\} & = \\
& = & \frac{1}{2 L N} \operatorname{Tr}\left\{\left(\boldsymbol{X}^{H} \boldsymbol{C}_{W}^{-1} \boldsymbol{X}\right)^{-1}\left(\boldsymbol{F}_{d} \boldsymbol{F}_{d}^{H}\right)^{-1}\right\} & = \\
& = & \frac{1}{2 L N} \operatorname{Tr}\{\boldsymbol{M} \boldsymbol{Q}\} & \geq \\
& \geq & \frac{1}{2 L N} \sum_{i=1}^{2 L} \mu_{i} \nu_{i}, &
\end{aligned}
$$

where $\mu_{i}$ and $\nu_{i}$ are the eigenvalues of $\boldsymbol{M}=\left(\boldsymbol{X}^{H} \boldsymbol{C}_{W}^{-1} \boldsymbol{X}\right)^{-1}$ and $\boldsymbol{Q}=\left(\boldsymbol{F}_{d} \boldsymbol{F}_{d}^{H}\right)^{-1}$ in non-decreasing and non-increasing order, respectively. The lower bound in (10) is achievable when matrices $M, Q$ are diagonal ([12, p. 249]).

By simple inspection ([4]), $M$ becomes diagonal when $\boldsymbol{X}_{2, L}=\mathbf{0}$ or else $\boldsymbol{q}=\underline{\mathbf{0}}$ and $E_{1}=E_{S}, E_{2}=0$. Moreover, $\boldsymbol{Q}=\left(\boldsymbol{F}_{d} \boldsymbol{F}_{d}^{H}\right)^{-1}$ is diagonal when pilot tones are equispaced, i.e., $i_{k}=(k-1) N / L, k=1,2, \ldots L$ and $N / L$ assumed to be integer ([7]). In this case, $\boldsymbol{Q}$ is expressed as $\boldsymbol{Q}=\frac{N}{L} \boldsymbol{I}_{L}$.

Based on these results, it turns out that (10) holds with equality, which after some straightforward manipulations is rewritten as

$$
f(\underline{\boldsymbol{p}}, \underline{\boldsymbol{e}})=\frac{1}{2 L^{2}}\left(\sum_{k=1}^{L} \frac{\gamma_{k}}{p_{k}}+\sum_{k=1}^{L} \frac{\delta_{k}}{e_{k}}+\sum_{k=1}^{L} \frac{\beta}{p_{k} e_{k}}\right)
$$

where $\gamma_{k}=\sigma_{D}^{2}+\sigma_{R}^{2} \theta_{k, R D}^{2}, \delta_{k}=\sigma_{D}^{2} \theta_{k, S R}^{2}$ and $\beta=\sigma_{D}^{2} \sigma_{R}^{2}$.

From (11), the minimization problem (9) is written as

$$
\min _{\underline{\boldsymbol{p}}, \underline{\boldsymbol{e}}} f(\underline{\boldsymbol{p}}, \underline{\boldsymbol{e}}), \text { s.t. } \sum_{k} e_{k}=E_{R}, \sum_{k} p_{k}=E_{S} .
$$

To the best of our knowledge there is no closed-form analytical solution to this minimization problem. In the following some interesting suboptimal schemes are presented, proceeding from the simpler to the more elaborated one.

\subsection{Case I}

When the pilot tones are equipowered at both the source and the relay, i.e., $p_{k}^{I}=E_{S} / L$ and $e_{k}^{I}=E_{R} / L$, the MSE in (11) is expressed as $M S E_{b o t h}=f\left(\underline{\boldsymbol{p}}^{I}, \underline{\boldsymbol{e}}^{I}\right)$. In this case, no further information is required by the source and the relay for training power allocation. This scheme will be used as a reference for the subsequent allocation schemes.

\subsection{Case II}

When only the source uses equipowered training symbols, i.e., $p_{k}^{I I}=E_{S} / L$, the $f\left(\underline{\boldsymbol{p}}^{I I}, \underline{\boldsymbol{e}}\right)$ is a function of $\underline{\boldsymbol{e}}$. By substituting $\boldsymbol{p}^{I I}$ in (12) and using Lagrange multipliers, the optimal power allocation at the relay turns out to be

$$
e_{k}^{I I}=\frac{\sqrt{\delta_{k}+\frac{L \beta}{E_{S}}}}{\sum_{j} \sqrt{\delta_{j}+\frac{L \beta}{E_{S}}}} E_{R}=\frac{\sqrt{\theta_{k, S R}^{2}+\frac{L \sigma_{R}^{2}}{E_{S}}}}{\sum_{j} \sqrt{\theta_{j, S R}^{2}+\frac{L \sigma_{R}^{2}}{E_{S}}}} E_{R}
$$

and the corresponding performance is given by $M S E_{\text {source }}=$ $f\left(\boldsymbol{p}^{I I}, \underline{\boldsymbol{e}}^{I I}\right)$. In this case, the relay requires knowledge of $\theta_{k, S R}^{2}$ 's and $\sigma_{R}^{2}$, which are second order statistical terms that are related only to the source-to-relay channel. As we will see shortly, this is an important difference with Case III requirements.

\subsection{Case III}

Similarly, if only the relay uses equipowered tones with $e_{k}^{I I I}=E_{R} / L$, then the optimal values at the source are

$$
p_{k}^{I I I}=\frac{\sqrt{\gamma_{k}+\frac{L \beta}{E_{R}}}}{\sum_{j} \sqrt{\gamma_{j}+\frac{L \beta}{E_{R}}}} E_{S}
$$

and the MSE is given by $M S E_{\text {relay }}=f\left(\underline{\boldsymbol{p}}^{I I I}, \underline{\boldsymbol{e}}^{I I I}\right)$. In this case, the source requires $\theta_{k, R D}^{2}$ 's, $\sigma_{R}^{2}$ and $\sigma_{D}^{2}$. Although, all these quantities are also second order statistical terms, they are more difficult to be acquired by the source, as they are mostly related to the relay-to-destination channel.

\subsection{Case IV}

Finally, an approach is described to minimize (12) if neither the source nor the relay use equipowered tones. First, (12) is minimized only with respect to the $p_{k}$ 's using Lagrange multipliers leading to

$$
p_{k}^{I V a}=\frac{\sqrt{\gamma_{k}+\frac{\beta}{e_{k}}}}{\sum_{j} \sqrt{\gamma_{j}+\frac{\beta}{e_{j}}}} E_{S} .
$$

By substituting (15) in (12), the optimization problem is expressed with repsect to the $e_{k}$ 's as follows

$$
\min _{e: \sum_{k} e_{k}=E_{R}} \frac{1}{2 L^{2} E_{S}}\left(\sum_{k} \sqrt{\gamma_{k}+\frac{\beta}{e_{k}}}\right)^{2}+\frac{1}{2 L^{2}} \sum_{k} \frac{\delta_{k}}{e_{k}} .
$$


To get a closed form solution from (16), we suggest to minimize an upper bound by utilizing Jensen's inequality. According to Jensen's inequality if a function $f(\cdot)$ is convex, then $f\left(\frac{\sum_{i} x_{i}}{L}\right) \leq \frac{\sum_{i} f\left(x_{i}\right)}{L}$ with equality if either all $x_{i}$ 's are equal or $f(\cdot)$ is linear. Applying this inequality to the first summation term in (16) and using Lagrange multipliers in the transformed problem yields

$$
e_{k}^{I V a}=\frac{\sqrt{\delta_{k}+\frac{L \beta}{E_{S}}}}{\sum_{j} \sqrt{\delta_{j}+\frac{L \beta}{E_{S}}}} E_{R}
$$

and the performance is $M S E_{\text {none }_{a}}=f\left(\boldsymbol{p}^{I V a}, \underline{\boldsymbol{e}}^{I V a}\right)$.

The same procedure can be followed if we start by minimizing first for $e_{k}$ 's and then for $p_{k}$ 's. The final results, in this case, are

$$
e_{k}^{I V b}=\frac{\sqrt{\delta_{k}+\frac{\beta}{p_{k}}}}{\sum_{j} \sqrt{\delta_{j}+\frac{\beta}{p_{j}}}} E_{R}, p_{k}^{I V b}=\frac{\sqrt{\gamma_{k}+\frac{L \beta}{E_{R}}}}{\sum_{j} \sqrt{\gamma_{j}+\frac{L \beta}{E_{R}}}} E_{S}
$$

and the performance is $M S E_{\text {none }_{b}}=f\left(\underline{\boldsymbol{p}}^{I V b}, \underline{\boldsymbol{e}}^{I V b}\right)$.

\section{DISCUSSION}

In the previous section, power allocation schemes for the training symbols were presented. The variety of the proposed schemes is due to the channel taps correlation assumption made in this work. Such an assumption is reasonable and leads to channels, whose $\theta_{k, S R}^{2}$ and $\theta_{k, R D}^{2}$ parameters take, in general, distinct values. It is thus clear that optimal power allocation for training should take into consideration the characteristics of the channels involved, and adapt the design according to each scenario. It should be noted that, all schemes fall into scheme $I$ when the channels are uncorrelated (in this case $\theta_{k, S R}^{2}=\theta_{S R}^{2}, \theta_{k, R D}^{2}=\theta_{R D}^{2}$ for all $k$ ). Moreover, using Jensen's inequality on the MSE results, it can be proven that

$$
\begin{aligned}
M S E_{\text {source }} \leq M S E_{\text {both }}, & M S E_{\text {relay }} \leq M S E_{\text {both }} \\
M S E_{\text {none }_{a}} \leq M S E_{\text {source }}, & M S E_{\text {none }_{b}} \leq M S E_{\text {relay }}
\end{aligned}
$$

These inequalities state that scheme $I V$ is always better than scheme II or III which, is superior than scheme I. Again, equalities are valid when the channels are uncorrelated.

Finally, we provide some simulations to verify our theoretical analysis. We assume that $\boldsymbol{C}_{i}$ 's are Toeplitz with first rows $\left[1, \rho^{1}, \ldots, \rho^{L_{i}-1}\right], 0 \leq \rho<1$ and $i \in\{S D, S R, R D\}$. We set $\rho=0.9, L_{S D}=16, L_{S R}=8, L_{R D}=9$ and $E_{S}=E_{R}=1$. In Fig. 2, the $M S E_{\text {both }}, M S E_{\text {source }}$ and $M S E_{\text {none }_{a}}$ versus the $S N R=10 \log _{10} \frac{E_{S}}{2 L \sigma_{D}^{2}}$ are plotted. It can seen that the theoretical results coincide with the experimental ones. Moreover, although not shown here, $M S E_{\text {both }}$ is independent of $\rho$. Finally, the performance of the schemes $I I, I I I$ and $I V$ tends very fast to that of scheme Case $I$, as $\rho$ goes to 0 (a similar observation was also made in [6]).

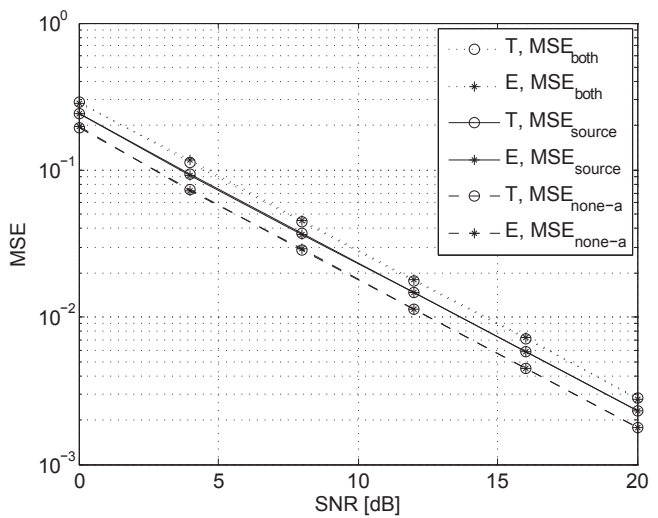

Fig. 2. (T)heoretical and (E)xperimental evaluation of three training power allocation schemes

\section{REFERENCES}

[1] A. Nosratinia, T. E. Hunter, and A. Hedayat, "Cooperative Communications in Wireless Networks," IEEE Comm. Mag., vol. 42, no. 10, pp. 74-80, Oct. 2004.

[2] K. Kim, H. Kim, and H. Park, "OFDM Channel Estimation for the Amply-and-Forward Cooperative Channel," in Proc. of IEEE VTC, 2007, pp. 1642-1646.

[3] K. Yan et al., "A low-complexity LMMSE channel estimation method for OFDM-based cooperative diversity systems with multiple Amplify-and-forward relays," EURASIP J. on Wireless Comm. and Networking, vol. 2008, no. 149803.

[4] C. Mavrokefalidis, A. A. Rontogiannis, and K. Berberidis, "Optimal Training Design and Placement for Channel Estimation in Cooperative networks," in Proc. of IEEE SPAWC, 2010.

[5] F. Gao, R. Zhang, and Y.-C. Liang, "Channel Estimation for OFDM Modulated Two-Way Relay Networks," IEEE Trans. on Sig. Proc., vol. 57, no. 11, pp. 4443-4455, Nov. 2009.

[6] B. Jiang, H. Wang, X. Gao, S. Jin, and K. Wong, "Preamblebased Channel Estimation for Amplify and Forward OFDM Relay Networks," in Proc. of IEEE Globecom, 2009.

[7] I. Barhumi, G. Leus, and M. Moonen, "Optimal Training Design for MIMO OFDM Systems in Mobile Wireless Channels," IEEE Trans. on Sig. Proc., vol. 51, no. 6, pp. 1615-1624, 2003.

[8] Z. Wang and G. B. Giannakis, "Wireless Multicarrier Communications: where Fourier meets Shannon," IEEE Signal Proc. Mag., vol. 17, no. 3, pp. 29-48, May 2000.

[9] R. U. Nabar, H. Bolcskei, and F. W. Kneubuhler, "Fading Relay Channels: Performance Limits and Space-Time Signal Design," IEEE J. on Sel. Areas in Comm., vol. 22, no. 6, pp. 1099-1109, Aug. 2004.

[10] E. Yoon, J. Hansen, and A. Paulraj, "Space-Frequency Precoding with Space-Tap Correlation Information at the Transmitter," IEEE Trans. on Com., vol. 55, no. 9, pp. 1702-1711, 2007.

[11] S. M. Kay, Fundamentals of statistical signal processing - Estimation theory, Prentice Hall, 1993.

[12] I. Olkin A. W. Marshall, Inequalities: Theory of Majorization and Its Applications, Academic Press, 1979. 